\title{
TIPIFICACIÓN DE LA SITUACIÓN ACTUAL, VENTAJAS Y RETOS DEL INGENIERO INDUSTRIAL DE LA UNIVERSIDAD DE COSTA RICA EN EL MERCADO LABORAL COSTARRICENSE
}

\author{
CURRENT SITUATION OF THE INDUSTRIAL ENGINEER \\ OF THE UNIVERSITY OF COSTA RICA, ADVANTAGES \\ AND CHALLENGES OF THE LABOR MARKET
}

José Francisco Roig Zamora ${ }^{1}$

Fecha de recepción: 6 de marzo de 2017 - Fecha de aceptación: 20 de junio de 2017

\begin{abstract}
Resumen
La creciente demanda de profesionales con un área de acción variable y flexible, con ideas innovadoras, en constante búsqueda de la mejora, ha llevado a un auge de la carrera de Ingeniería Industrial. No obstante, existe un segmento de la población conformado por futuros estudiantes universitarios, padres de familia, tutores, asesores vocacionales o encargados de brindar ayuda en el proceso de selección de carrera, que desconocen o no entienden en su totalidad, que es la ingeniería industrial, cuáles son sus campos de acción y cuál es el rol de este profesional en el mercado laboral. Por lo tanto, se pretende en este artículo, mediante una encuesta sistemática, recolectar la voz de las 441 personas que han cursado la carrera ofrecida por la Escuela de Ingeniería Industrial de la Universidad de Costa Rica entre el año 2007 hasta el 2014. Entre los principales hallazgos se destaca que el 28\% de los encuestados tardan cinco años en concluir la carrera, el resto tardó seis años o más. Se identificó que la carrera brinda flexibilidad para que los estudiantes trabajen (36\% de los estudiantes encuestados). Los principales puestos ejercidos por los encuestados graduados son de "Ingeniero" (26\%), "Analista" (21\%) y "Jefe" (14\%) y dicha oferta laboral se presenta en su mayoría en el sector privado (89\%). El estudio permite facilitar la comprensión del público meta acerca de la carrera y promover su estudio al brindar un acercamiento de la realidad y las oportunidades existentes para los ingenieros industriales.

Palabras claves: carrera universitaria, especialización laboral, ingeniería industrial, mercado laboral, situación actual.
\end{abstract}

\begin{abstract}
The growing demand for professionals with a variable and flexible action area, innovative ideas and constantly seeking for improvement, has led to a boom of the Industrial Engineering career. However, there is a segment of the population comprised of students, parents, vocational counselors or people responsible for providing assistance in the selection of career to high school students, that do not know or understand fully, what industrial engineering is, what are its fields of action and the role of this professional in the labor market. Therefore, it is intended in this article, through a systematic survey, to collect the voice of the 441 people who have passed from 2007 until 2014 the career offered by the School of Industrial Engineering at the University of Costa Rica. Among the main findings of the study, 28\% of respondents took five years to complete the career, the rest took six years or more. It was identified that the career offers the opportunity to work and study (36\% of the surveyed students). The main positions exercised by the surveyed graduates are "Engineer" (26\%), "Analyst" (21\%) and "Boss" (14\%). Also, the labor supply is presented mostly in the private sector (89\%). Through the study, it was possible to
\end{abstract}

1 Escuela de Ingeniería Industrial, Facultad de Ingeniería, Universidad de Costa Rica, roigjose@gmail.com 
facilitate the target audience information regarding the career and promote their interest by having and approach of the available opportunities for these professionals.

Key Words: College career, current situation, industrial engineering, job specialization, labor market.

\section{Introducción}

Dentro del contexto universitario y educativo, sin duda alguna, una de los momentos más importantes en la vida de un profesional es el de la toma de decisiones para la escogencia de una carrera. Son precisamente las personas que vivieron lo anterior, las que cuentan con información valiosa para recomendar a futuros decidores, acerca de factores claves a tomar a consideración, esto a partir de la experiencia de vida.

Esta investigación tiene como eje central la recopilación sistemática de información clave de estudiantes y profesionales ingenieros industriales de la Universidad de Costa Rica. A partir de lo que se espera indagar lo concerniente a los siguientes objetivos:

- Determinar una de las variables más importantes que impacta el proceso de obtención del título de licenciatura de los estudiantes de la carrera de ingeniería industrial, correspondiente a la complejidad de graduación. A su vez, dotar de esta información a la población interesada por su relación con el proceso de escogencia de la carrera (sea, futuro estudiante universitario o padres de familia, tutores, asesores vocacionales o encargados en proceso de ayudar en dicha selección).

- Investigar la flexibilidad y posibilidad que brinda la carrera a los estudiantes, para trabajar mientras se cursa la carrera y dotar al tomador de decisiones de esta información en caso de que sea relevante en su situación personal.

- Entender la realidad de la carrera en términos del proceso de incorporación al mercado laboral, con el objetivo de determinar qué tanta demanda ofrece el mercado y dotar de esta información al estudiante en sus distintas etapas de madurez (sea, una persona en proceso de selección de carrera, un estudiante de primer ingreso a la universidad, un estudiante avanzado en sus estudios o un estudiante recién graduado).

- Determinar las principales áreas y tipos de empresas en las cuales están trabajando los ingenieros industriales graduados de la UCR. Lo anterior con el objeto de brindar un acercamiento de la amplitud del mercado laboral disponible, así como las diferentes áreas en las que puede incursionar un ingeniero industrial.

- Determinar el desarrollo académico promedio de un ingeniero industrial posterior a su graduación de licenciatura. Esto con el fin de identificar la existencia de un mercado con demanda en especialistas con mayor grado académico y por lo tanto, tener un parámetro de la importancia de continuar estudiando posterior a la obtención del título de licenciatura.

- Determinar el desarrollo profesional promedio alcanzado por los ingenieros industriales en relación con sus años de experiencia laboral; así como los principales rangos que éstos ejercen en el mercado laboral según la especialización en que trabajan. Esto con el fin de brindar al estudiante en diferentes etapas de madurez, un acercamiento de la realidad del mercado laboral, las posibilidades de crecimiento que existen en este, así como las diferentes oportunidades dependiendo de la rama por la que se incline.

- Entender la situación actual del mercado laboral de la ingeniería industrial respecto a variables macro importantes a tomar en cuenta para la escogencia de un trabajo, tales como: las áreas prioritarias de especialización laboral de los graduados, el rango de horas promedio de la jornada laboral, así como las oportunidades de trabajo dependiendo de si se incursiona en el sector público o privado. Con el objeto de brindar información clave y actualizada acerca de la experiencia laboral 
de los ingenieros y las oportunidades existentes, y apoyar la toma de decisiones de la población interesado, así como aquellos estudiantes que desean estudiar en el área de ingeniería pero aún no eligen la rama específica.

- Determinar el grado de participación de la mujer entre los estudiantes de la carrera y determinar cuáles son las tendencias que se han presentado en los años recientes, con el objetivo de entender la realidad actual de la ingeniería industrial y el grado de potencial y oferta que ofrece actualmente.

\section{Referente teórico}

\section{Contextualización teórica de la Ingeniería industrial}

El Instituto de Ingenieros Industriales y de Sistemas (IISE) establece que la ingeniería industrial es aquella que se encuentra relacionada con el diseño, mejora e instalación de sistemas integrados conformados por personas, materiales, información, equipos y energía. Se basa en el conocimiento especializado y habilidades en las ciencias matemáticas, físicas y sociales, así como los principios y la metodología del análisis y diseño, para especificar, predecir y evaluar los resultados que se obtienen de los sistemas. (IISE, 2016) La Escuela de Ingeniería Industrial (EII) de la Universidad de Costa Rica (UCR) complementa lo anterior, al definir que un ingeniero (a) industrial es el/la profesional que se ocupa de aumentar la eficiencia y eficacia de dichos sistemas. (EII, 2016)

Debido a la complejidad de los sistemas mencionados, el ingeniero industrial debe contar con conocimiento y habilidades en una gran variedad de disciplinas, la capacidad de trabajar correctamente con gente y una amplia perspectiva sistémica. Esto les permite mejorar procesos sistemáticos por medio del análisis estadístico, comunicación interpersonal, diseño, planeación, control de calidad, manejo de las operaciones, simulación y resolución de problemas. (Oregon State University, 2016)

A su vez, el Tecnológico de Monterrey define que un profesional en ingeniería industrial y de sistemas es aquel que cuenta con "una base sólida en matemáticas y ciencias naturales, está especializado en la optimización de recursos (personas, maquinaria, materiales, información, energía, dinero y tecnología) de una organización, para la fabricación de productos o la prestación de servicios; cuenta con una visión integral de negocios y tiene como referencia el desarrollo sostenible". (Tecnológico de Monterrey, 2016)

Entre los aspectos más distintivos de la carrera de Ingeniería Industrial, se tiene la flexibilidad que ofrece, esto de acuerdo con la Universidad de Illinois. De manera que conforme las empresas adoptan filosofías de gestión enfocadas hacia la productividad continua y mejora de la calidad para sobrevivir en mercados cada vez más competitivos, los ingenieros(as) industriales resultan ser los profesionales adecuados, al ser especialistas en dichos enfoques. (Universidad de Illinois, 2016)

\section{Contextualización teórica de los ámbitos de la Ingeniería industrial}

Los ingenieros industriales determinan las formas más efectivas de utilizar factores básicos de producción como personas, máquinas, materiales, información y energía para hacer un producto o proveer de un servicio. Para ello, estudian cuidadosamente los requerimientos del producto, para luego diseñar con ayuda de métodos y modelos matemáticos sistemas de manufactura e información que alcancen dichos requerimientos. También desarrollan sistemas de control de la gestión para apoyar la planeación financiera y análisis de costos y a su vez diseñar la planeación de producción y sistemas de control para coordinar actividades y asegurar la calidad del producto. Además diseñan o mejoran sistemas de distribución física de productos terminados y servicios y determinan las locaciones más eficientes para las plantas. (The State University of New York, 2016) 
Para lograr lo anterior, la malla curricular de la carrera se conforma de distintos ámbitos. La EII de la UCR define que éstos son los siguientes:

- "Planeación y programación de la producción

- Control estadístico de la calidad

- Implementación sistemas de gestión de la calidad, ambientales, de salud ocupacional y de responsabilidad social

- Análisis, rediseño y mejora de procesos

- Diseño de la estructura organizacional

- Sistema de manejo de materiales y almacenamiento en bodegas

- Conceptualización de sistemas de información

- Distribución y localización de instalaciones

- Análisis financiero y evaluación de proyectos de inversión

- Gestión de proyectos

- Diseño del trabajo: medición de tiempos, estudios de métodos, aplicación de la ergonomía

- Gestión estratégica y de desempeño

- Diseño de sistemas de manufactura y robótica

- Diseño de productos y servicios"

(EII, 2016)

De manera que a partir de las diferentes herramientas obtenidas de los ámbitos de la carrera, ésta provee de prácticas de negocio más eficientes y rentables y aumenta los niveles de servicio a los clientes y la calidad. Los ingenieros industriales hacen que el ambiente de trabajo sea más seguro, rápido, fácil y más gratificante. Buscan ofrecer métodos por medio de los cuales las empresas pueden analizar sus procesos e intentar realizar mejoras, manteniendo un enfoque en la optimización, es decir, "hacer más con menos". (Universidad de Illinois, 2016)

La Universidad del Norte de Barranquilla Colombia, clasifica los campos de acción de la carrera en los siguientes (Universidad del Norte Barranquilla Colombia, 2016):

- Gestión de Operaciones: Incluye los ámbitos relacionados con planeación, programación y control de la producción, simulación, diseño, normalización y estandarización de métodos de trabajo.

- Gestión de la Calidad: Se relaciona con el "diseño e implementación de sistemas de aseguramiento, garantía y control de calidad". (Universidad del Norte Barranquilla Colombia, 2016)

- Logística y Distribución: Engloba la "administración de procesos de compras de material, gestión de almacenes y bodegas, sistemas de inventarios, distribución y transporte de los productos", determinación de la "localización, tamaño y diseño de las instalaciones operativas". (Universidad del Norte Barranquilla Colombia, 2016)

- Seguridad Industrial y Gestión Ambiental: Integración de los factores seguridad y ambiente en la cadena productiva, de manera que permita identificar, evaluar y diseñar estrategias para evitar que se materialicen riesgos específicos de los distintos procesos.

- Gestión Económico - Administrativa: Incluye el estudio de los mercados que atiende la empresa y nuevos por incursionar, desarrollo de productos, análisis de alternativas de inversión, elaboración de presupuestos, gestión de proyectos de inversión, planificación estratégica del negocio, entre otros.

- Gestión del Talento Humano: Gestión bajo el enfoque de competencias, a través de la definición de roles y responsabilidades, valoración del desempeño, etc. 


\section{Contextualización acerca del rol de la Ingeniería Industrial en el mercado laboral}

La ingeniería industrial se puede desarrollar en cualquier lugar donde se realice un proceso o exista un sistema. (EII, 2016) Ya sean empresas de comercialización, educación, logística, manufactura y/o servicios.

La Universidad Nacional de Ingeniería (UNI) de Perú establece que un ingeniero industrial se debe caracterizar por ser capaz de liderar en equipos de trabajo, buscar formas de mejorar los distintos procesos de las empresas, diseñar y plantear soluciones, realizar mejoras con base al costo - beneficio para la empresa, planificar, organizar, dirigir y controlar procesos productivos de la organización, trabajar en conjunto con grupos multidisciplinarios hacia la mejora organizacional, conducir sus gestiones con responsabilidad social y ética, entre otros. (Universidad Nacional de Ingeniería, 2016)

Otras responsabilidades definidas por el Occupational Outlook Handbook del Bureau of Labor Statistics de Estados Unidos, corresponden a las siguientes (Bureau of Labor Statistics, 2015):

- Revisión de horarios de producción, especificaciones técnicas, flujos de proceso y otra información relacionada para entender métodos aplicados y actividades que se desarrollan en la producción y prestación de servicios.

- Idear cuanto producir (componentes y producto final) o entrega de servicios a un máximo de eficiencia.

- Desarrollar sistemas de control de la gestión para realizar la planeación financiera y de esta forma los análisis de costos resulten más eficientes.

- Llevar a cabo procedimientos de control de calidad para resolver problemas de producción o minimizar costos.

- Diseñar sistemas de control para asegurar que los productos cumplen con los estándares de calidad.

- Coordinar con clientes las especificaciones de los productos, con los vendedores acerca de las ventas, con administración del personal acerca de las capacidades de producción y con el personal acerca del estatus de proyectos.

Por lo que, la Universidad del Norte en Colombia establece que el perfil profesional de un graduado de la carrera debe ser una persona "creativa, dinámica y segura; con capacidad para afrontar y liderar el cambio. Debe estar dispuesto y motivado para afrontar un medio globalizado y competitivo en la búsqueda del mejoramiento continuo y la optimización de los recursos, alcanzando la máxima competitividad". (Universidad del Norte - Colombia -, 2016) Además debe contar con habilidades de pensamiento crítico, capacidad de escuchar y entender las necesidades de sus clientes (internos y/o externos), contar con alta capacidad de habla, escritura y resolución de problemas, para así idear soluciones viables para las empresas. (Truity, 2016)

\section{Contextualización teórica de la técnica de encuesta}

Existen dos tipos principales de encuestas, las aplicadas de forma escrita denominadas como cuestionarios y las que se aplican de forma oral, conocidas como entrevistas. Se diferencian en que la primera puede ser aplicada a distancia, sin necesidad de que coincidan los horarios del encuestador y la persona objetivo. Mientras que el segundo tipo requiere de contacto físico presencial. (Barrantes R, 2000, p. 186)

Entre las principales partes de la realización y aplicación de una encuesta, se tiene el diseño adecuado de los datos que se requieren para lograr los objetivos de la investigación. Puesto que si se comenten errores en las etapas de observación y recolección de datos, los resultados se encontrarán sesgados y puede llevar al investigador a conclusiones incorrectas. 
Una de las mejores actitudes que puede adoptar un investigador ante los datos, es ser crítico, pues según Gómez, esta es una de las características que distinguen al investigador de ser bueno o no. Para ello, éste debe conocer las fuentes de datos, así como su interpretación, pues le permitirá garantizar la asertividad de las conclusiones que propondrá en su estudio. (Gómez, M., 1999)

También resulta importante evaluar la fuente de información, para ello, entre los puntos que se deben tomar en cuenta se tienen los siguientes:

- Definir si es posible obtener los datos por medio de un muestreo o si resulta necesario realizar un censo de la totalidad de la población.

- Garantizar la parcialidad del proceso, así como que no se da un conflicto de intereses que pueda introducir sesgo en los resultados.

- Planificar correctamente la secuencia de preguntas, así como el debido procedimiento.

Entre los métodos existentes para llevar a cabo una encuesta se tienen la observación, la entrevista, el correo electrónico, entre otros. Se resalta que si bien la encuesta es uno de los métodos más efectivos de investigación, también resulta en uno de los más difíciles y costos. Esto debido a la necesidad de coordinar la presencia y disponibilidad de aplicación tanto del entrevistado como del entrevistador en un mismo momento y espacio, lo cual requiere de los recursos financieros necesarios para financiar el estudio.

Gracias a las evoluciones tecnológicas y la mejor aceptación social de los medios tecnológicos, ha tenido auge el método electrónico de encuesta mediante un cuestionario. De manera que a través de aplicaciones web es posible que el investigador genere una encuesta mediante un cuestionario y lo envíe al correo electrónico del encuestado, el cual puede contestarla desde la comodidad de su casa y en el momento que desee. Se podría decir que esto lleva a un aumento de la participación de la población meta y probablemente la calidad de respuestas.

Por otro lado, se resalta que el cuestionario es un instrumento conformado por una serie de preguntas, las cuales deben ser resueltas sin intervención del investigador. Por lo que resulta importante que la formulación de las mismas sea la adecuada para obtener información pertinente, válida y confiable para el estudio. En donde una formulación adecuada se refiere a que las preguntas no excluyan preguntas claves o que por el contrario, se incluyan preguntas no relevantes que le generen cansancio y desmotivación al entrevistado. (Barrantes R, 2000, p. 188) M., 1999)

Varias limitantes del método que deben ser consideradas, corresponden a las siguientes: (Gómez,

- El cuestionario debe contar con instrucciones claras. Las preguntas del mismo deben redactarse y ordenarse con una secuencia que garantice la facilidad de resolución. Esto busca evitar que las personas no contesten o contesten de forma incorrecta las preguntas incluidas en el estudio.

- La población meta de la encuesta debe tener la capacidad y familiaridad con el método electrónico.

- Debido a que el porcentaje de "no respuesta" de encuestas es alto, Gómez propone diseñar y planificar envíos de invitaciones y recordatorios a los participantes para que éstos colaboren con contestar el cuestionario.

Dado que el método de investigación requiere de la colaboración de un grupo de personas, se resalta la importancia de un cauteloso diseño de la encuesta, para que ésta resulte atractiva de resolución para el encuestado. Por lo general se recomienda explicar el contexto de la investigación, así como la importancia de la opinión propia de cada encuestado, esto con el objeto de motivar a que el encuestado comparta su opinión honesta y transmita la información requerida. (Barrantes R, 2000, p. 188)

A su vez, Gómez (Gómez, M., 1999) propone que el eje primario del diseño de la investigación debe ser el problema a investigar claramente definido, seguido por los objetivos específicos. Además, 
define que los estudios fundamentalmente son de naturaleza exploratoria o descriptiva. Por lo tanto, la construcción del cuestionario puede variar, ya sea mediante entrevista abierta, por teléfono o correo electrónico, además puede incluir preguntas cerradas y abiertas; de manera que corresponde a una naturaleza más de exploración.

Con respecto a la muestra de la población meta, esta debe ser seleccionada de acuerdo con el método y situación de la investigación. En el presente caso, la encuesta se dirige hacia los estudiantes que han aprobado al menos el 50\% de la carrera entre el año 2007 hasta el 2014 (lógicamente incluye un alto porcentaje de estudiantes que ya aprobaron toda la carrera desde el 2007), lo cual corresponde a 441 personas.

Una vez claro lo anterior, de acuerdo con la teoría de investigación por medio de encuesta, los pasos a ejecutar posterior al diseño del cuestionario, corresponde al procesamiento de los datos obtenidos, análisis e interpretación de los mismos, preparación del informe y la generación de conclusiones. (Gómez, M., 1999).

\section{Procedimiento metodológico}

Una fuente de información muy válida para conocer acerca de la situación de los ingenieros industriales son aquellos estudiantes actuales y graduados de la carrera. Dichos estudiantes y profesionales que pueden dar a conocer acerca de su vida universitaria, así como su ejercicio profesional en las empresas, resultan en una útil fuente primaria de información.

Por lo tanto, se procedió a diseñar un cuestionario que permitiese recolectar información actualizada acerca de la situación de los ingenieros industriales y así alcanzar los objetivos del estudio.

Como parte de la población meta de este cuestionario se definieron los estudiantes que han aprobado al menos el 50\% de la carrera entre el año 2007 hasta el 2014, lo cual corresponde a 441 personas. Dichos estudiantes se clasificaron en aquellos que actualmente se dedican solo a sus estudios, estudiantes activos y que trabajan y estudiantes graduados y trabajando.

El tipo de encuesta utilizado correspondió a la encuesta por medio de un cuestionario "en línea". Dado que la entrevista personal no resulta viable para el investigador debido a factores de tiempo y disponibilidad de atender a toda la población meta deseada.

Para realizar un correcto diseño del cuestionario por aplicarse, se llevó a cabo una sesión de discusión y análisis con un grupo de estudiantes actuales de la carrera y graduados. A partir de ello, se determinaron los distintos puntos de interés y se priorizaron a partir de su importancia relativa. Esto permitió una recolección inicial de datos para la posterior confección del cuestionario de la encuesta.

Con base en la población meta definida, se tomó la decisión de realizar un censo, pues se contó con información de contacto personal, como correos electrónicos y números telefónicos. Por lo tanto, se aplicó la encuesta por el método electrónico, específicamente mediante una aplicación de encuesta en línea libre y de código abierto llamada LimeSurvey. Esta utiliza una base de datos de MySQL y es distribuida bajo la Licencia Pública General GNU. La aplicación corresponde a un software basado en un servidor web que permite a los usuarios utilizar una interfaz web para desarrollar y publicar encuestas en línea, recopilar respuestas, crear estadísticas generales y exportar los datos resultantes a otras aplicaciones (como Excel). Además, la aplicación permitió confeccionar correos electrónicos personalizados para invitar a las personas específicas a participar, mensajes de bienvenida, recordatorios de participación y agradecimientos posteriores a la participación.

Por lo tanto, luego de la sesión de discusión antes mencionada, se confeccionó una versión inicial del cuestionario en la aplicación. Se planificó la correcta secuencia de preguntas, así como la respectiva consulta bibliográfica, para evitar la imparcialidad del proceso o el conflicto de intereses del encuestado al completarla.

Se realizó una prueba piloto por medio del envío de la encuesta a diez personas de las distintas clasificaciones antes mencionadas, sin comunicarles que correspondía una prueba. Luego de obtener sus 
respuestas, se contactaron nuevamente de forma directa para obtener retroalimentación del cuestionario y el mecanismo empleado para la aplicación del mismo. Esto permitió identificar oportunidades de mejora al instrumento, específicamente en el orden que se plantearon las preguntas, así como modificaciones de la configuración de la plataforma de LimeSurvey.

La prueba piloto resultó de suma importancia antes del lanzamiento masivo a la población de 441 personas, ya que permitió robustecer su diseño, así como evitar identificar errores posteriores al lanzamiento de la encuesta final. Además, debido a que la encuesta "en línea" puede resultar en una herramienta que dificulta la recolección de información, al no aplicarse de forma presencial. Resulta vital que esté bien afinada, para aprovechar la única oportunidad de que el encuestado llene la encuesta.

Una vez concluidas las fases previas, se obtuvo un cuestionario con preguntas cerradas que surgieron a partir del conocimiento del investigador, así como el aporte de la sesión inicial y la prueba piloto. En los casos aplicables, se brindó al encuestado la opción de responder: "otros", para que digitara su respuesta específica, sino la encontraba la adecuada dentro de las opciones brindadas. Por medio del uso de reglas lógicas en la configuración de la aplicación de la encuesta, se evitó que los encuestados se saltaran preguntas. Además, a partir de su condición, algunas preguntas se mostraban y otras no. De manera que se impidió obtener información errónea y no confiable para el estudio.

El cuestionario contempló las siguientes dos secciones:

- Información General de la Población de Estudio: Con el objeto de perfilar al encuestado en términos de experiencia laboral, segmento en el que labora y perfil académico.

- Condiciones Laborales de la Población Meta del Estudio: Con el fin de perfilar la situación actual laboral de la población de estudiantes que ya aprobaron al menos la mitad de la carrera.

Una vez realizado el lanzamiento oficial de la encuesta, se programaron recordatorios cada diez días hábiles luego de la invitación de participación o el último recordatorio realizado. La encuesta estuvo disponible por un período de dos meses y medio durante el año 2015.

La plataforma sobre la cual se confeccionó la encuesta permitió generar estadísticas generales de los resultados obtenidos. Sin embargo, con el objeto de analizar con mayor profundidad la información obtenida, se confeccionó una herramienta programada en Excel que permitió extraer otros resultados de interés.

\section{Sistematización de los resultados obtenidos con la innovación}

A partir de la investigación realizada, fue posible categorizar los resultados en: la situación de la ingeniería industrial en términos de la vida académica y laboral en la etapa de estudiante universitario y la situación de los ingenieros industriales graduados y en actual ejercicio profesional de la carrera.

\section{Situación del estudiante de ingeniería industrial}

Con el fin de determinar una de las variables más importantes que impacta el proceso de selección de carrera, se investigó acerca de la dificultad de obtener el título de licenciatura. Para ello se preguntó de forma directa a los encuestados acerca de la complejidad de graduación en términos del tiempo que tardaron en concluir la carrera. El $28 \%$ de los encuestados ya graduados dio a conocer que durante su época de estudiante, tardaron cinco años en obtener su título, es decir, concluir con los cursos de la carrera, el trabajo comunal universitario, las horas conferencia solicitadas por la EII, así como el desarrollo y presentación de sus tesis para optar por el título de licenciatura. Por otro lado, el 35\% de los encuestados tardó seis años, el 22\% tardó siete años en concluir con sus estudios, un 5\% tardó ocho años, un 7\% tardó nueve años, mientras que el 3\% restante no lo detalló. De manera que se puede observar que la mayoría de encuestados duró al menos un año más de lo establecido en el actual plan de estudios 
de la EII, en donde esta define que en diez ciclos lectivos, es decir cinco años, los estudiantes concluyen con todos los cursos de la licenciatura.

A raíz de lo anterior, se buscó indagar acerca de la flexibilidad que brinda la carrera en términos de horarios de clases, carga académica de los distintos cursos, proyectos en empresa, así como la existencia de un mercado laboral para estudiantes próximos a graduarse, entre otros factores de interés para aquellos que evalúan la posibilidad de estudiar y trabajar. Para ello, se preguntó a los estudiantes que participaron en la encuesta si trabajan. Se obtuvo que el $64 \%$ de los estudiantes encuestados no trabajan, mientras que un $36 \%$ si estudia y trabaja. De los estudiantes que no trabajan, el $67 \%$ justificó que se debe a una decisión de dedicarse por completo a sus estudios, el 17\% mencionó que no ha encontrado una opción laboral que le permita trabajar y estudiar, mientras que el 15\% restante no detalló su razón específica.

Una vez que se determinó que si existen posibilidades de trabajar y estudiar en la carrera, a partir del 36\% de los estudiantes encuestados que se encuentran en dicha condición, surgió la curiosidad de tipificar las empresas en las que trabajan, con respecto al sector, área, así como la carga laboral que deben asumir los estudiantes en términos de horas de la jornada laboral. Como resultado de este estudio se obtuvo que el $84 \%$ trabaja en empresas del sector privado, un $14 \%$ se encuentra en el sector público y un $2 \%$ no detalló el sector. A su vez, cabe destacar que el $47 \%$ de estudiantes que trabajan, lo hacen en jornadas de 40 horas a la semana, mientras que un $40 \%$ trabaja más de 40 horas por semana, un $7 \%$ trabaja de 21 a 30 horas por semana, un $5 \%$ trabaja de 11 a 20 horas y por ende un $2 \%$ trabaja menos de 10 horas por semana. De manera que esto permite identificar la carga laboral que asumen los estudiantes que deciden también trabajar, así como que entre las desventajas se presenta que en su mayoría deben trabajar tiempos completos, y en menor cantidad existen posibilidades de medios o cuartos de tiempos a la semana. Además, se resalta que si bien un bajo porcentaje de los encuestados consideró que no ha encontrado opciones laborales factibles para su condición, se determina que si existe un mercado laboral disponible para los estudiantes de ingeniería industrial.

De la muestra de los estudiantes que trabajan también se obtuvo que la mayoría lo hace en el área de Mejora de Procesos (19\%), seguido por el área de Calidad y Proyectos con 14\% de los encuestados cada uno, un 12\% trabaja en Planning (Demanda, Producción o Materiales), luego un 7\% trabaja en Compras/Importaciones, otro 7\% en Recursos Humanos, un 5\% en Manufactura, un 3\% en Sistemas de Información y Finanzas (en cada uno), en las áreas de Almacenes y Bodega, Distribución, Ventas e Inteligencia Comercial trabaja un $2 \%$ en cada uno y por último un $9 \%$ no trabaja en ninguno de los anteriores.

Por otro lado, con el fin de entender la realidad de la carrera en términos del proceso de incorporación al mercado laboral, se preguntó de forma directa a los encuestados, tanto actuales estudiantes como graduados de la carrera, como evalúan el grado de dificultad para encontrar trabajo como ingeniero industrial. De ello se identificó que el 50\% consideró que es "Ni fácil, ni difícil", un $42 \%$ calificó el proceso como "Fácil" y un $8 \%$ estableció que le parece "Difícil". Por lo que es posible identificar que el $92 \%$ de los encuestados considera que la carrera presenta demanda de nuevos profesionales y en general no resulta difícil obtener trabajo en el área.

\section{Situación del graduado de ingeniería industrial}

Una vez que los estudiantes se gradúan como ingenieros industriales, resulta de interés conocer acerca de las principales áreas y tipos de empresas para las cuales laboran.

De manera que resulte posible identificar, no sólo que los graduados en esta carrera pueden obtener un trabajo de forma fácil, sino los tipos de empresas en las que trabajan, las diferentes áreas en las que pueden incursionar en dichas empresas y en cuáles de estas existe mayor cantidad de demanda.

De los encuestados graduados y que actualmente trabajan en ingeniería industrial, fue posible priorizar los tipos de empresas en las que estos laboran, éstas corresponden a: "Servicios" con un 35\% 
de los encuestados, seguido por un $27 \%$ que trabaja en empresa de "Manufactura", un $17 \%$ trabaja en "Comercialización", un 11\% en "Logística", un 2\% en "Educación" y un $8 \%$ en ninguno de los anteriores. A su vez, las principales áreas en las que laboran los ingenieros industriales dentro de éstos tipos de empresas corresponden a: "Proyectos" con un 17\% de los encuestados, seguido por "Planning (Demanda, Producción o Materiales)" con un 15\%, un 14\% de los encuestados labora en el área de "Mejora de Procesos", un $10 \%$ trabaja en "Calidad", un 6\% en "Sistemas de Información", un 5\% en "Inteligencia Comercial". En las áreas de "Manufactura" y "Almacenes y Bodega" trabaja un $4 \%$ en cada una, en "Compras / Importaciones" y "Mercadeo" trabaja un 3\% en cada una, en "Distribución", "Finanzas" y "Ventas" trabaja un 2\% en cada una, en "Recursos Humanos" trabaja un $1 \%$ y un $13 \%$ en ninguna de las anteriores.

Una vez determinados los tipos de empresa y áreas de mayor preponderancia en el mercado laboral de la carrera, se busca determinar el desarrollo académico promedio de un ingeniero industrial posterior a su graduación de licenciatura, con el fin de identificar si dicho mercado demanda especialistas de este tipo. Para ello, se preguntó a los encuestados el año en que se graduaron de la universidad con el título de licenciatura y el mayor grado académico obtenido hasta el momento de aplicación de la encuesta (año 2014). A partir de ello, fue posible obtener que el $77 \%$ de los encuestados únicamente permanece con el título de licenciatura, un $22 \%$ optó por el título de maestría, un $1 \%$ optó por un Doctorado, mientras que otro $1 \%$ cuenta con otro grado académico. Para aquellos que mantienen únicamente la licenciatura, se destaca que el $82 \%$ tiene entre uno y cuatro años de haberse graduado y el $18 \%$ restante tiene entre cinco y siete años. Por otro lado, de aquellos que optaron por la maestría, el $80 \%$ tienen entre cuatro y siete años de haberse graduado de la licenciatura y el $20 \%$ tiene entre un año y tres años. Por lo tanto, se nota un punto de cambio a los cuatro años de graduados de licenciatura, pues la mayoría se inclina en este punto por desarrollarse a nivel académico, al optar por el grado de maestría. Lo que probablemente se encuentra correlacionado con el hecho de que a esta etapa de la vida profesional ya el ingeniero crece en criterio profesional y busca desarrollarse más.

También resulta de interés conocer acerca del desarrollo profesional que alcanzan los ingenieros industriales una vez que se encuentran inmersos en el mercado laboral. Para ello se preguntó a los encuestados acerca del puesto actual que ejercen en las empresas, así como la cantidad de años que tienen trabajando, esto como indicador de madurez profesional posterior a incursionar en el mercado laboral. De ello se obtuvo que, el 26\% de los encuestados labora en el puesto de "Ingeniero", el 22\% trabaja como "Analista", 15\% trabaja en el puesto de "Jefe", el 10\% es "Coordinador", el $8 \%$ es "Gerente", el 6\% es "Especialista", un 4\% tiene el puesto de "Encargado", un 1\% es "Director" y un $8 \%$ no es ninguno de los anteriores.

A continuación se detalla por puesto de trabajo de los encuestados, la cantidad de años que tienen trabajando, con el objeto de identificar si existe diferencia entre los puestos que asumen ingenieros industriales que trabajan con menos de un año de graduados hasta cinco años o más de trabajar.

- Ingeniero: El 35\% de los encuestados que laboran en este puesto tienen cinco años o más de trabajar, el 28\% tiene entre dos y cuatro años, el 27\% tiene entre uno y dos años y el $10 \%$ tiene menos de un año.

- Analista: El 43\% de analistas tienen entre dos y cuatro años de trabajar posterior a graduarse de licenciados, un $22 \%$ tiene cinco años o más, otro $22 \%$ tiene entre uno y dos años, mientras que un $12 \%$ tiene menos de un año de graduado.

- Jefe: El 64\% de los jefes tienen más de cinco años de trabajar, el 30\% tiene entre dos y cuatro años, el $3 \%$ tiene entre uno y dos años y otro $3 \%$ tiene menos de un año.

- Coordinador: Un 43\% de ingenieros industriales que trabajan como coordinadores tienen cinco años o más de graduados y de trabajar, otro $43 \%$ tiene entre dos y cuatro años y un $13 \%$ tiene menos de un año. 
- Gerente: El 63\% de los gerentes cuentan con cinco años o más de trabajar posterior a su graduación, un $32 \%$ tiene entre dos y cuatro años de experiencia y un 5\% entre uno y dos años.

- Especialista: El 46\% de los especialistas tiene cinco años o más de experiencia, un 38\% tiene entre dos y cuatro años y un $15 \%$ tiene menos de un año.

- Encargado: El 44\% de encargados tiene entre dos y cuatro años de experiencia, un $22 \%$ cuenta con cinco años o más de experiencia, a su vez, otro $22 \%$ tiene entre uno y dos años y un $11 \%$ tiene menos de un año.

- Director: El 67\% de los encuestados que ejercen como directores tienen cinco años o más de experiencia y un $33 \%$ tiene entre dos y cuatro años.

De lo anterior es posible obtener datos relevantes de la realidad del mercado laboral que se encuentra disponible para los ingenieros industriales. En donde existe variedad de puestos a los que puede aspirar el graduado y que a su vez existen oportunidades de crecimiento, por lo general después de cuatro a cinco años de trabajar y contar con experiencia. Se resalta que el puesto en el que mayor cantidad de encuestados ya graduados trabajan es el de "Ingeniero", una vez que cuentan con cinco años o más de experiencia. Además, puestos como el de "Gerente" y "Director" no fueron alcanzados por ninguno de los encuestados con menos de un año de experiencia y por un porcentaje bajo (5\% correspondiente a un encuestado) fue alcanzado entre uno y dos años, los demás casos todos contaron como mínimo con más de dos años de experiencia y en su mayoría cinco años o más. Cabe destacar que si bien la ventana de análisis del estudio puede ocasionar sesgo en los resultados, se consideró de importancia la inclusión de lo obtenido, para proveer al tomador de decisiones de las variables necesarias para su interpretación y análisis. Por otro lado, se identifica que el puesto de "Analista" es alcanzado por la mayoría de encuestados entre dos y cuatro años de trabajar, al igual que el puesto de "Encargado". Así como que, el puesto en el cual se ubican la mayor cantidad de encuestados con menos de un año de graduados es el de "Especialista" seguido por "Coordinador".

Con el fin de obtener mayor detalle acerca de la situación actual del mercado laboral en el cual se encuentran inmersos los ingenieros industriales, se preguntó a los encuestados, tanto estudiantes actuales de la carrera como graduados, acerca de las áreas prioritarias de especialización laboral de acuerdo con su percepción de la demanda del mercado. De ello se obtuvo que el $45 \%$ de los encuestados considera que el área con mayor demanda del mercado laboral resulta la "Planeación y Manejo de Inventario", seguido por un $24 \%$ que considera que es el "Servicio al Cliente", un $18 \%$ opinó que es el "Abastecimiento (Compras)", un 11\% "Gestión de Centro de Distribución y Bodegas" y un 2\% opinó que "Transporte". A su vez, se preguntó a los encuestados que ya trabajan, con cual de dichas áreas se relaciona más su trabajo actual, de lo que se obtuvo que el 30\% labora en el área de "Servicio al Cliente", seguido por un 24\% que trabaja en "Planeación y Manejo de Inventario", un 11\% trabaja en "Abastecimiento (Compras)", un 8\% en "Gestión de Centro de Distribución y Bodegas" y finalmente, la minoría correspondiente al 5\% trabaja en "Transporte". Por lo que se nota que las áreas de servicio al cliente e inventarios se encuentran entre los principales caminos por los que el graduado debe optar, pues existe mayor demanda del mercado. Mientras que áreas como las de transportes cuentan con menor oferta para los mismos.

Además, se cuestionó a los encuestados acerca del sector en el que se encuentran las empresas para las que trabajan, De ello se obtuvo que el $89 \%$ de los encuestados que trabajan lo hacen empresas del sector privado, un $11 \%$ labora en empresas del sector público, mientras que un $1 \%$ trabaja en un sector diferente a los anteriores. Por lo que es posible dar a conocer a la población interesada, que en su mayoría las opciones de oferta laboral se encuentran en el área privada, aunque el área pública también es una opción. Como se nota, se presenta una coincidencia con el caso de los estudiantes que deciden trabajar y estudiar.

Por otro lado, se identificó que el 66\% de ingenieros industriales que trabajan lo hacen en jornadas de más de 40 horas por semana, un $31 \%$ trabaja jornadas de 40 horas semanales, un $2 \%$ trabaja de 11 
a 20 horas por semana, un $1 \%$ trabaja de 21 a 30 horas y otro $1 \%$ trabaja menos de 10 horas por semana. Por lo tanto, de forma similar al caso de los estudiantes que deciden trabajar, las jornadas laborales por lo general rondan entre las 40 horas semanales y más (jornada típica del mercado costarricense).

Con el objeto de ahondar en lo anterior, se buscó tipificar el mercado en términos de las áreas de trabajo y los puestos más populares que alcanzan los graduados según el área. Posteriormente, realizar un análisis de los puestos de trabajo antes identificados y las respectivas jornadas laborales que conllevan. A continuación se presentan los resultados obtenidos para la tipificación de las áreas y puestos de trabajo:

- Proyectos: El 31\% de encuestados que labora en el área de proyectos funge como "Ingeniero", el $21 \%$ como "Coordinador", un 14\% como "Analista", otro 14\% como "Jefe", como "Encargado", "Especialista", "Gerente" y "Director" trabaja en cada uno un 3\% y un 7\% trabaja en el área de proyectos, pero en ninguno de los puestos anteriores.

- Planning (Demanda, Producción o Materiales): Un 28\% de los ingenieros industriales que trabajan en esta área tiene el puesto de "Analista", otro 28\% tiene el puesto de "Ingeniero", un $16 \%$ es "Especialista", un 12\% es "Coordinador", un 8\% es "Jefe", un 4\% "Encargado" y otro $4 \%$ no ejerce ninguno de los puestos incluidos en el estudio.

- Mejora de Procesos: El 57\% de los encuestados que trabajan en mejorar procesos tienen el puesto de "Ingeniero", un 13\% es "Analista", un 9\% es "Especialista", otro 9\% es "Jefe" y como "Encargado", "Coordinador" y "Director" trabaja en cada uno un $4 \%$.

- Calidad: De los ingenieros que trabajan en calidad, $41 \%$ tiene el puesto de "Ingeniero", el 24\% es "Jefe", un 12\% es "Gerente" y como "Analista", "Encargado", "Coordinador" trabaja un 6\% en cada uno. Mientras que otro 6\% que trabaja en dicha área no ejerce ninguno de los puestos mencionados en el estudio.

- Sistemas de Información: El 60\% que trabaja en dicha área es "Analista", como "Coordinador", "Ingeniero" y "Director" trabaja en cada uno un 10\%. Así como que otro $10 \%$ no ejerce ninguno de los puestos anteriores.

- Inteligencia Comercial: El 33\% de los encuestados es "Coordinador", un 22\% es "Analista", a su vez, otro $22 \%$ es "Gerente" y como "Ingeniero" y "Jefe" trabaja en cada área un $11 \%$ de los encuestados.

- Manufactura: El 83\% de encuestados que trabajan en esta área son "Jefe", mientras que el 17\% es "Ingeniero".

- Almacenes y Bodega: Un 50\% de los ingenieros industriales en dicha área fungen como "Ingeniero", como "Encargado" y "Jefe" trabaja en cada área un 17\%. Así como que el 17\% restante no ejerce ninguno de los puestos incluidos en el estudio.

- Compras / Importaciones: El 40\% de los encuestados es "Gerente", un $20 \%$ es "Especialista", otro $20 \%$ es "Jefe" y el $20 \%$ restante no ejerce ninguno de los puestos mencionados en el estudio.

- Mercadeo: El 60\% tiene el puesto de "Analista", un 20\% es "Coordinador" y otro 20\% es "Jefe".

- Distribución: Un 50\% de los ingenieros industriales que trabajan en distribución son "Gerente", un $25 \%$ es "Ingeniero" y otro $25 \%$ no ejerce ninguno de los puestos incluidos en el estudio.

- Finanzas: El 50\% es "Especialista" en finanzas, un 25\% es "Jefe" y otro $25 \%$ es "Gerente".

- Ventas: Los puestos ejercidos en la presente área corresponden a "Analista", "Jefe" y "Gerente", cada uno con un $33 \%$ de los encuestados.

- Recursos Humanos: Para el caso de recursos humanos, un 50\% de los encuestados son "Analista" y otro $50 \%$ ejerce el puesto de "Especialista".

Con respecto a la jornada laboral de los encuestados que se graduaron y trabajan, se nota una tendencia hacia jornadas de 40 horas semanales o más de 40 horas a la semana (con predominancia de ésta última), para aquellos que ejercen los puestos de "Analista", "Encargado", "Coordinador", "Jefe", 
"Gerente" y "Director". Mientras que se identificó que en el puesto de "Especialista" e "Ingeniero", existen encuestados que laboran de 11 a 20 horas por semana, con un 5\% y 9\% respectivamente.

De manera que fue posible obtener información clave acerca de la experiencia laboral de los ingenieros industriales, en donde estos pueden ejercer gran variedad de puestos dependiendo del área en que se encuentren las respectivas empresas, así como el número de años de experiencia laboral con que cuentan. Dichas empresas son en su mayoría del sector privado y los puestos exigen en su mayoría jornadas completas, con únicamente puestos como el de "Especialista" e "Ingeniero", cuya jornada para algunos de los casos de los encuestados es de menor cantidad de horas a la semana. Además, a partir de los resultados obtenidos es posible presentar a aquellos estudiantes en proceso de selección de carrera que desean estudiar en el área de ingeniería pero aún no eligen la rama específica, la gran variedad de puestos, tipos de empresas y áreas de especialización laboral que son disponibles y demandadas por el mercado para los ingenieros industriales.

Por último pero no menos importante, otra variable que resulta de interés estudiar, es el aumento de la presencia de la mujer en el ambiente laboral y académico de la ingeniería industrial en la última década y media. Para ello, se buscó determinar el grado de participación de la mujer entre los estudiantes y graduados de la carrera en diferentes períodos entre el rango de años del 2000 al 2014. Esto debido a que corresponde al mínimo y máximo de años en que la muestra de personas que participaron en el estudio ingresó a la carrera. A groso modo, se obtuvo que de la totalidad de encuestados que continúan estudiando, un $37 \%$ son mujeres mientras que un $63 \%$ son hombres. Así como que de todos los graduados involucrados en el estudio, un $42 \%$ son ingenieras industriales, mientras que un $58 \%$ son ingenieros. Con el fin de identificar con mayor detalle si existe un aumento en la participación, se segmentó el rango de años en tres períodos: estudiantes y graduados entre el 2000 y 2004, estudiantes y graduados entre el 2005 y 2009 y estudiantes y graduados entre el 2010 y 2014. A continuación se presentan los resultados obtenidos:

- $\quad$ Período 2000 a 2004

- Estudiantes Mujeres: 2,7\%

- Estudiantes Hombres: 10,8\%

- Graduadas Mujeres: 35,1\%

- Graduados Hombres: 51,4\%

- $\quad$ Período 2005 a 2009

- Estudiantes Mujeres: 8,8\%

- Estudiantes Hombres: $18,7 \%$

- Graduadas Mujeres: 30,6\%

- Graduados Hombres: $41,9 \%$

- Período 2010 a 2014

- Estudiantes Mujeres: 40,8\%

- Estudiantes Hombres: $57,2 \%$

- Graduadas Mujeres: $1 \%$

- Graduados Hombres: $1 \%$

A partir de lo anterior, es posible observar el aumento en la presencia de las mujeres estudiantes de ingeniería industrial con el paso de los años. En el caso del período del 2000 al 2004, la proporción de estudiantes es de 1:4 (una mujer por cada cuatro hombres), del 2005 al 2009 hay un aumento, pues pasa a ser 1:2. Para los años 2010 a 2014, la relación es de 5:7. En el caso de los graduados entre los años 2000 a 2004, la relación es de un $68 \%$ de mujeres por cada $100 \%$ de hombres, es decir, una relación 13:19. Para los graduados entre 2005 y 2009 la proporción es de 73\% mujeres por cada 100\% (es decir, una relación 49:67) y por último, para el período de 2010 a 2014, la relación es 1:1. Por lo tanto, de forma similar a los estudiantes de la carrera, en el caso de los graduados, la presencia de la mujer ha aumentado con el paso de los años. De manera que es posible observar que si bien no existe una predominancia de mujeres, si existe un mercado laboral disponible que ofrece espacio, tanto para hombres como mujeres. 


\section{Conclusiones generales}

A partir del estudio realizado, se presentan las siguientes conclusiones:

- Tras investigar el grado de complejidad de graduación de la carrera en términos del número de años que se tarda en obtener el título universitario, se determinó que el $28 \%$ de los sujetos incluidos en el estudio duraron el tiempo definido por el plan de estudios de la EII correspondiente a cinco años. Mientras que el $72 \%$ restante tardó seis años o más.

- La carrera brinda flexibilidad para que los estudiantes trabajen, además el mercado presenta oferta de puestos de trabajo para personas en dicha condición. Por lo que resulta posible que los estudiantes opten por la opción de trabajar. Esto se determinó a partir de estudiar la condición de los estudiantes que participaron en la encuesta, en donde el 64\% solo estudia y el $36 \%$ aún no se ha graduado de la carrera pero si tiene un trabajo relacionado con el área. Se resalta que únicamente un $17 \%$ de los estudiantes encuestados que solo estudian justificaron que no trabajan pues no han encontrado una opción laboral que le permita realizar ambas acciones.

- A partir de la situación del 36\% de estudiantes encuestados que estudian y trabajan, se identificó que las principales oportunidades de trabajo se encuentran en el sector privado, esto a partir de que el 84\% de los estudiantes que se encuentran en dicha condición están ubicados en este sector. Por otro lado, un $14 \%$ trabaja para el sector público y un $2 \%$ no trabaja en ninguno de los anteriores. Las jornadas laborales asumidas por el $87 \%$ de los encuestados que estudian y trabajan se encuentran entre 40 horas semanales y más de 40 horas por semana (47\% y $40 \%$ respectivamente). Además, los principales puestos asumidos por la mayoría de estudiantes que trabajan corresponde a: Mejora de procesos (19\%), Calidad (14\%), Proyectos (14\%) y Planning de demanda, producción o materiales (12\%). Por lo que se determina la existencia de un mercado laboral para ingenieros industriales no graduados, en las empresas del sector privado que en su mayoría solicitan a los estudiantes que trabajen tiempos completos y en menor cantidad existen posibilidades de medios o cuartos tiempos a la semana.

- El proceso de incorporación al mercado laboral de la ingeniería industrial es calificado por el 92\% del total de encuestados como "ni fácil, ni difícil" y "fácil" con porcentajes respectivos de 50\% y $42 \%$. Por lo que es posible determinar que la carrera presenta demanda de nuevos profesionales y en general a estos no les resulta difícil obtener trabajo en el área.

- El mercado laboral disponible para los ingenieros industriales graduados se califica como amplio al ofrecer oportunidades en diferentes tipos de empresas. El 90\% de los encuestados graduados que trabajan, lo hacen en empresas de Servicios (35\%), Manufactura (27\%), Comercialización (17\%) y Logística (11\%), el 10\% restante trabaja en Educación y en áreas distintas de las anteriores. A su vez, a partir de la situación actual de los encuestados graduados y que trabajan, se determinó que las áreas de trabajo principales corresponden a: Proyectos (con 17\% de los encuestados que trabajan), Planning (Demanda, Producción o Materiales) (15\%), Mejora de Procesos, (14\%) Calidad (10\%), Sistemas de Información (6\%), Inteligencia Comercial (5\%), Manufactura (4\%), Almacenes y Bodega (4\%), Compras / Importaciones (3\%) y Mercadeo (3\%). El 19\% restante trabaja en las áreas de Distribución, Finanzas, Ventas, Recursos Humanos y en áreas distintas de las anteriores.

- Se determinó con respecto al desarrollo académico, que el 22\% de los encuestados graduados optaron por el título de maestría y el $80 \%$ de estos tienen entre cuatro y siete años de haberse graduado de la licenciatura. Por lo que se identifica en dicha etapa una motivación por parte de los encuestados de seguir estudiando, al obtener un título de mayor grado académico.

- A partir del estudio de la situación del encuestado graduado de la carrera, fue posible analizar el desarrollo profesional promedio alcanzado por los mismos. Los principales puestos ejercidos por los graduados encuestados corresponden a los siguientes: Ingeniero (26\%), Analista (22\%), Jefe $(15 \%)$, Coordinador (10\%), Gerente (8\%), Especialista (6\%), Encargado (4\%) y por último Director 
(1\%). El 8\% restante de encuestados graduados no detalló su puesto de trabajo. Se resalta que si bien el puesto de "Ingeniero" es en el que trabaja mayor cantidad de graduados, este es alcanzado por la mayoría después de cinco años o más de experiencia. Los puestos de "Gerente" y "Director" no fueron alcanzados por ninguno de los encuestados con menos de un año de experiencia, únicamente un 5\% (de los encuestados que trabajan y son gerentes) alcanzó el puesto de "Gerente" entre uno y dos años de experiencia laboral. Los demás contaron como mínimo con más de dos años de experiencia y en su mayoría cinco años o más. Por otro lado, en su mayoría el puesto de "Analista" es alcanzado entre dos y cuatro años de trabajar, al igual que el puesto de "Encargado". El puesto en el cual se ubican la mayor cantidad de encuestados con menos de un año de graduados es el de "Especialista" seguido por "Coordinador".

- A partir del estudio de distintos períodos de años de la última década y media, fue posible determinar el aumento en la presencia de la mujer entre los estudiantes y graduados de la carrera. Específicamente en el caso de los estudiantes, se identificó el aumento de una relación 1:4 (una mujer por cada cuatro hombres) entre los años 2000 y 2004, hacia una relación 1:2 entre 2005 y 2009 y 5:7 entre 2010 y 2014. En el caso de los graduados, la proporción aumentó de $68 \%$ mujeres por cada $100 \%$ de hombres entre el 2000 y 2004, hacia un $73 \%$ entre el 2005 y 2009, hasta alcanzar una relación 1:1 entre el 2010 y 2014. Por lo que se identifica la existencia de un mercado laboral disponible que ofrece espacio, tanto para hombres como mujeres.

- A partir de la investigación de variables macro importantes a tomar en cuenta para la escogencia de trabajo, como lo son las áreas prioritarias de especialización laboral de los graduados, el rango de horas promedio de la jornada laboral, así como las oportunidades de trabajo dependiendo de si se incursiona en el sector público o privado, se determinó lo siguiente:

- Las principales áreas de especialización laboral por las que deben optar los graduados corresponden a "Servicio al Cliente" y "Planeación y Manejo de Inventario", mientras que el área de menor oferta es "Transportes". Esto fue determinado a partir de la percepción de los encuestados (actuales estudiantes y graduados) de la demanda del mercado laboral, así como de la relación de las áreas de especialización con los trabajos actuales ejercidos por los graduados.

- La mayoría de opciones de oferta laboral se encuentra en empresas del sector privado, el 89\% de los encuestados que trabajan, lo hacen en este tipo de empresas. A diferencia de un $11 \%$ que labora en empresas del sector público y el $1 \%$ restante que trabaja en un sector diferente de los anteriores. Se resalta que se presenta una segmentación proporcional en el caso de los estudiantes que trabajan.

- La mayoría de trabajos disponibles para los graduados solicitan entre 40 horas semanales y más de 40 horas semanales. Esto se determinó a partir de la situación del $97 \%$ de los encuestados que trabajan, puesto que el $66 \%$ de los encuestados que trabajan lo hacen en jornadas de más de 40 horas semanales y el $31 \%$ en jornadas de 40 horas semanales. Además, se destaca que se identificaron únicamente casos en los puestos de "Especialista" e "Ingeniero" en los que las jornadas de algunos encuestados resultaron ser de menor cantidad de horas a la semana, específicamente de 11 a 20 horas por semana, con porcentajes respectivos de $5 \%$ y $9 \%$ de los encuestados que trabajan.

- El área de "Proyectos" es en el que mayor cantidad de graduados trabajan (31\% de los encuestados que trabajan) y a su vez, presenta mayor variedad de puestos por los que pueden optar los ingenieros industriales, desde "Ingeniero" y "Coordinador" hasta "Gerente" y "Director". El área de "Planning (Demanda, Producción o Materiales) corresponde a la segunda área en la que trabaja la mayor cantidad de graduados (28\%), en esta, los principales puestos son de "Analista" e "Ingeniero". Entre las áreas que presentan menor variedad de puestos se tiene "Manufactura" con los puestos de "Jefe" e "Ingeniero". Así como "Distribución", con los puestos de "Gerente" e "Ingeniero". 


\section{Referencias}

Barrantes Gómez, Miguel. (1999). Elementos de Estadística Descriptiva. Editorial Euned. Universidad Estatal a Distancia. Costa Rica.

Barrantes Echavarría, Rodrigo. (2000). Investigación: un camino al conocimiento. Editorial Euned. Universidad Estatal a Distancia. Costa Rica.

Bureau of Labor Statistics (2015) Occupational Outlook Handbook: Industrial Engineers. Fecha de consulta: 30 de Marzo del 2016. URL: http://www.bls.gov/ooh/architecture-and-engineering/ industrial-engineers.htm\#tab-2

Escuela de Ingeniería Industrial (s.f.) Ingeniería Industrial es: ¿Qué es un (a) Ingeniero (a) Industrial?. Fecha de consulta: 22 de Marzo del 2016. URL: http://www.eii.ucr.ac.cr/carrera/ingindustriales.html

Institute of Industrial Engineers (s.f.) About IIE: What is industrial engineering? (IEE oficial definition). Fecha de consulta: 23 de Marzo del 2016. URL: https://www.iienet2.org/details.aspx?id=282

Oregon State University (s.f.) Mechanical, Industrial and Manufacturing Engineering: What Do Industrial Engineers Do? Fecha de consulta: 29 de Marzo del 2016. URL: http://mime.oregonstate.edu/ what-do-industrial-engineers-do

Tecnológico de Monterrey (s.f.) Ingeniero Industrial y de Sistemas: Descripción. Fecha de consulta: 23 de Marzo del 2016. URL: http://www.itesm.mx/wps/wcm/connect/itesm/tecnologico+de+monterrey/ carreras+profesionales/areas+de+estudio/ingenieria+y+ciencias/iis

The State University of New York, University at Buffalo. (s.f.) Industrial and Systems Engineering: Why Be an Industrial Engineer? Fecha de consulta: 30 de Marzo del 2016. URL: http://www.ise.buffalo.edu/undergraduate/why_be

Truity (s.f.) Industrial Engineer: Important Qualities. Fecha de consulta: 31 de Marzo del2016. URL: http://www.truity.com/career-profile/industrial-engineer

Universidad de Illinois (s.f.) Industrial \& Enterprise Systems Engineering: What is Industrial Engineering? Fecha de consulta: 30 de Marzo del 2016. URL: http://ise.illinois.edu/sites/default/files/ whatisie.pdf

Universidad del Norte. Barranquilla, Colombia (s.f.) Ingeniería Industrial: Campos de acción. Fecha de consulta: 30 de Marzo del 2016. URL: http://www.uninorte.edu.co/web/ingenieria-industrial/ perfiles

Universidad Nacional de Ingeniería (s.f.) Competencias del Ingeniero Industrial. Fecha de consulta: 07 de Junio del 2016. URL: http://fiisvirtual.uni.edu.pe/sistemas/competencia/ 